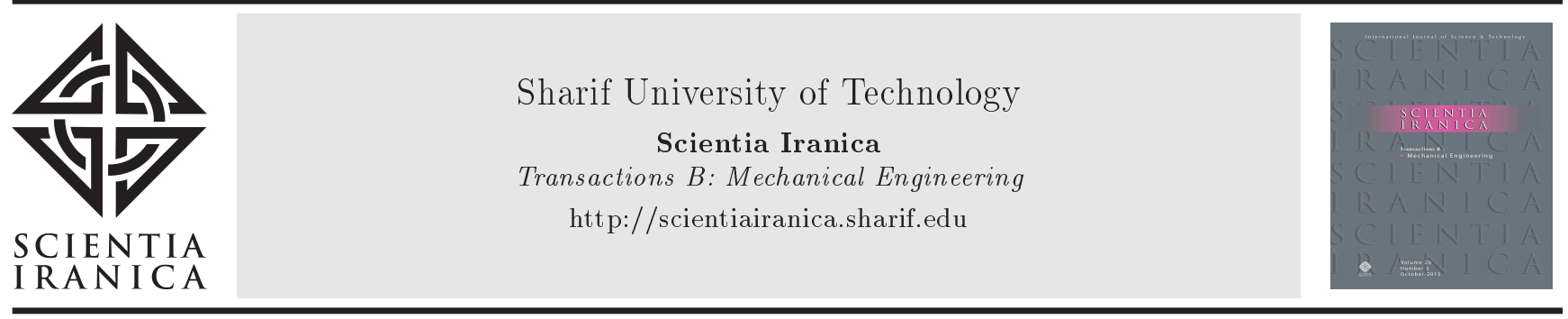

\title{
A quasi-3D modified strain gradient formulation for static bending of functionally graded micro beams resting on Winkler-Pasternak elastic foundation
}

\author{
M. Gholami and M. Alizadeh* \\ School of Mechanical Engineering, Iran University of Science and Technology, Tehran, 16846, Iran.
}

Received 9 December 2019; received in revised form 9 March 2021; accepted 17 May 2021

\author{
KEYWORDS \\ Modified strain \\ gradient theory; \\ Quasi-3D theory; \\ Two-parameter elastic \\ foundation; \\ Size dependent \\ behavior; \\ Functionally graded \\ materials.
}

\begin{abstract}
This paper presents the bending analysis of simply supported Functionally Graded (FG) size-dependent beams based on modified strain gradient theory. Shear and normal deformations are considered in displacement field according to hyperbolic shear deformation theory. Governing equations and corresponding boundary conditions for FG micro beam are derived utilizing principle of minimum total potential energy. MoriTanaka homogenization scheme and the classical rule of mixture are used for prediction of material properties through the thickness. Effects of Winkler-Pasternak elastic foundation parameters are studied at different side-to-thickness ratios. Effects of different aspect ratios, elastic foundation parameters, power law gradient indexes, and different loading conditions are investigated. The efficiency and accuracy of the presented model is demonstrated against the existing results in particular cases.
\end{abstract}

(C) 2022 Sharif University of Technology. All rights reserved.

\section{Introduction}

Beams on the order of microns and sub-microns are unavoidable parts of modern world and widely used as sensors [1], actuators [2], atomic force microscopes [3], and in Micro/Nano Electro-Mechanical Systems (MEMS/NEMS). A number of experiments have proved that results of classical continuum approach are not acceptable when the size decreases and this is the deficiency of classical theory for capturing size effects [4], which can account for the size dependencies of micro and nano structures.

The development of higher order theories dates back to 19th century by works of Piola [5] and

*. Corresponding author. Tel./Fax: +98 21 73228958 E-mail address: ma_alizadeh@iust.ac.ir (M. Alizadeh)

doi: $10.24200 /$ sci.2021.55000.4019
Cosserat and Cosserat [6]. Several approaches have been introduced in order to consider micro-/nanoscale effects, among which the continuum mechanics approach provides greater simplicity and efficiency in predicting size effect behavior than Molecular Dynamic (MD) approach [7]. Mindlin [8] considered secondorder gradients of deformation and introduced general higher-order theory with five length scale parameters. Couple Stress Theory (CST) is presented in the works of Toupin [9], Mindlin and Tiersten [10], and Koiter [11]. In this theory, higher order rotation gradients are incorporated. Yang et al. [12] modified the classical CST and developed Modified Couple Stress Theory (MCST) by enforcing the couple stress tensor to be symmetric, in which only one length scale parameter was included.

Subsequently, Fleck and Hutchinson [13,14] extended and reformulated the first version of Mindlin theory and renamed it Strain Gradient Theory (SGT), 
in which the deformation gradient tensor is composed of one rotation gradient and two independent stretch gradient tensors. Lam et al. [15] utilized the higher-order equilibrium equation suggested by Yang et al. [12] and presented Modified Strain Gradient Theory (MSGT). The presented theory included three material length scale parameters to characterize dilatation, deviatoric and symmetric rotation gradient tensors. MCST can be achieved as a special case of MSGT by including only a rotation tensor.

MSGT formulation has gained much attention recently and has been widely employed by researchers. Dal [16] analyzed Euler-Bernoulli micro gold beams and demonstrated the accuracy of the results in comparison to the existing experiment tests. Ashoori and Mahmoodi [17] presented a geometric nonlinear formulation for analysis of thick plates. Chu et al. [18] elaborated general MSGT for static bending and natural frequency analysis of Functionally Graded Material (FGM) Euler-Bernoulli piezoelectric nanobeams. They used volume fraction function for FGM nanobeams and concluded that material distribution function, flexoelectric coefficient ratio, and span-to-depth ratio had considerable effects on the electromechanical response of nanobeams. Tai et al. [19] investigated free vibration of Functionally Graded (FG) hexagonal beryllium crystal micro plates by using Iso-Geometric Analysis (IGA). They employed MSGT in conjunction with Higher order Shear Deformation Theory (HSDT) in order to consider shear effects. Another study on micro plates using IGA was performed by Farzam and Hassani [20]. They analyzed bending and buckling responses of FGM micro plates under mechanical and thermal loads. They assumed that the materials with temperature-dependent properties and several rise patterns were explored. They also presented the margins for material length scale ratio at which scale effects were negligible. Cornacchia et al. [21] solved the static bending of laminated Kirchhoff nano plates and examined different stacking sequences and loading profiles. Recently, some studies have employed IGA analysis and conducted further experiments based on the nonlocal and HSDT for free vibration and bending analysis of FG plates [22], geometrically nonlinear transient analysis of FGM nanoplates [23,24], and static and free vibration analyses of porous FG nanoplates [25].

Farzam and Hassani [26] examined the bending, buckling, and free vibration behaviors of in-plane FG porous microplates. They also investigated thermal and mechanical buckling analyses of FG carbon nanotube reinforced composite nanoplates based on MCST and IGA [27] and investigated the accuracy and efficiency of the proposed model for different dimensional and power indexes. Effective computational optimization approaches based on Eringen's nonlocal elasticity and four variables refined plate theory were introduced for optimal design [28] and porosity-dependent analysis of FG sandwich nanoplates [29]. Zhao et al. [30] proposed a nonlinear size-dependent formulation for bending and vibration analysis of nanobeams. They used MSGT and generalized differential quadrature method to derive and discretize nonlinear governing equations. They found that both strain gradient and flexoelectric coupling had considerable impact on nonlinear behavior. Moreover, inclusion of surface effects diminished the flexoelectric response. Zanoosi [31] discussed the free vibration of porous FG micro beams under thermo-mechanical loading using MSGT. He further explored the effects of different parameters such as thermal loading, slender ratio, and gradient index for different beam theories.

Classical Beam Theory (CBT) is the simplest theory for beam analysis, but the results are limited to thin beams. Rotary inertia and shear effects were first reported by Timoshenko [32] with some improvements over CBT, and several studies [33] were conducted using First-order Shear Deformation Theory (FSDT). Higher order shear deformation theories have been introduced to cover shortcomings of the latter theory, i.e., stress free surface condition. These theories assume power series expansion in thickness coordinate and offer acceptable precision compared to existing theories. In HSDT, conventional higher order terms take into consideration shear effects and compensate the above-mentioned drawbacks of FSDTs. Reddy [34] considered a third-order polynomial expansion for displacement field and studied the bending analysis of isotropic and anisotropic beams. Afterwards, different types of HSDTs were implemented $[35,36]$. Ninh and Bich analyzed the nonlinear vibration [37] and nonlinear torsional buckling [38] of Eccentrically Stiffened (ES) FG toroidal shell segments in thermal environment with the geometrical nonlinearity and surrounded by an elastic medium based on the classical shell theory.

Quasi-3D theories are presented as another extension of shear deformation theories by introducing thickness stretching effects in transverse deflection function. These theories can be used by a unified formulation presented by Carrera [39], and they were consequently developed by Demasi [40]. Karamanli and Vo [41] investigated the flexural behavior of FG micro beams by employing quasi-3D formulation and MCST. They employed finite element method and studied different boundary conditions and length scale parameters. Benahmed et al. [42] used a hyperbolic quasi-3D theory for bending and free vibration analysis of FG plates. They also studied the effects of elastic foundation parameters. Nguyen et al. [43] investigated the free vibration and buckling analysis of FG sand wich beams for various boundary conditions by employing Ritz-type quasi-3D solution. Farzam and Hassani [44] 
developed a new HSDT for static and free vibrations and buckling analysis of FG plates with in-plane and through-thickness stiffness variations. Farzam-Rad et al. [45] studied static and free vibrations of FG and sandwich plates based on IGA and quasi-3D theory. A quasi-3D shear deformation plate theory combined with MCST theory was employed by Thai et al. [46]. They studied the impact of length-to-thickness ratios, weight fraction values, and material length scale-tothickness ratios on free vibration and buckling behavior of multilayer FG graphene platelet-reinforced composite microplates.

In order to simulate the interaction between beams and elastic foundations, various models have been introduced. The simplest model is Winkler [47] elastic foundation that assumes a series of vertical independent springs. Pasternak [48] offered a more general model that accounts for the shear interaction between Winkler springs. Atmane et al. [49] studied the effects of porosity and thickness stretching on the static and dynamic responses of FG micro beams resting on elastic foundation. Lee et al. [50] studied the static bending response of simply supported plates. The material properties assumed to vary according to exponential power law, and effects of two-parameter Pasternak elastic foundation were investigated. $\mathrm{Li}$ et al. [51] employed MSGT combined with HSDT in order to study the buckling behavior of organic solar cells. The model rested on Winkler-Pasternak elastic foundation and Galerkin procedure was employed to determine critical buckling loads. They also considered thermal effects and stated that mechanical buckling of the organic solar cell was more critical than thermal buckling. Ninh et al. [52] investigated the nonlinear vibration of $\mathrm{W}-\mathrm{Cu}$ sandwich shell that contained heavy water under thermo-mechanical loads. They concluded that the nonlinear response of sandwich shells was significantly influenced by geometrical parameters, material, temperature, and elastic foundation.

Zeighampour et al. [53] studied the wave propagation in viscoelastic single-walled carbon nanotubes resting on a viscoelastic Pasternak foundation. They employed Hamilton's principle for deriving governing equations and Kelvin-Voigt model for expressing the viscoelastic property. Several studies have been performed on responses of beams and plates resting on elastic foundation and under moving loads [54], micro beams conveying fluid [55], and interaction with viscoelastic foundations [56].

Bich and Ninh performed several studies on static and dynamic analyses of FGM toroidal shell segments including nonlinear dynamic buckling [57], nonlinear vibration in an external thermal environment containing fluid [58] and nonlinear buckling and post-buckling behavior of shells surrounded by elastic foundation [59]. They examined the effects of imperfection, fluid, and geometrical and material parameters on the nonlinear behavior of shell segments.

In the present study, a simply supported sizedependent beam resting on elastic foundation is considered. The displacement field is based on quasi-3D theory. Static bending responses for different geometrical and foundation parameters were investigated using MSGT. To the best of the authors' knowledge, effects of foundation parameters on bending behavior of FGM size-dependent beams based on quasi-3D approach (accounting for $\varepsilon z z \neq 0$ ) in conjunction with MSGT have not been studied before. Quasi 3-D theories are subject to lower computational costs than 3-D theories; moreover, they offer acceptable accuracies compared to existing approaches. Possible applications of the present model might include curvature sensors, structural health monitoring, and implantable BioMEMS devices.

\section{Problem formulation}

\subsection{Quasi-3D displacement field}

The beam under study is assumed to have a rectangular cross-section with length $L$ along $x$ direction, and it rests on a two-parameter elastic foundation, as depicted in Figure 1. Right-handed Cartesian coordinate system is adopted. Width $b$ and thickness $h$ lie along $y$ and $z$ directions, respectively. The FG size-dependent beams are generally composed of two different materials on the top and bottom surfaces. Gradual changes in material properties for beam from bottom to top surface were estimated according to power law index. Here, the Young modulus and Poisson's ratio are assumed to vary through thickness according to the classical rule of mixture and Mori-Tanaka scheme.

The displacement field for quasi-3D HSDTs is commonly considered as follows:

$$
\begin{aligned}
& u(x, z)=u_{0}(x)-z \frac{\mathrm{d} w_{b}(x)}{\mathrm{d} x}-f(z) \frac{\mathrm{d} w_{s}(x)}{\mathrm{d} x}, \\
& w(x, z)=w_{b}(x)+w_{s}(x)+g(z) w_{z}(x),
\end{aligned}
$$

where $u_{0}, w_{b}$, and $w_{s}$ are axial displacement, bending, and shear parts of transverse displacements, respec-

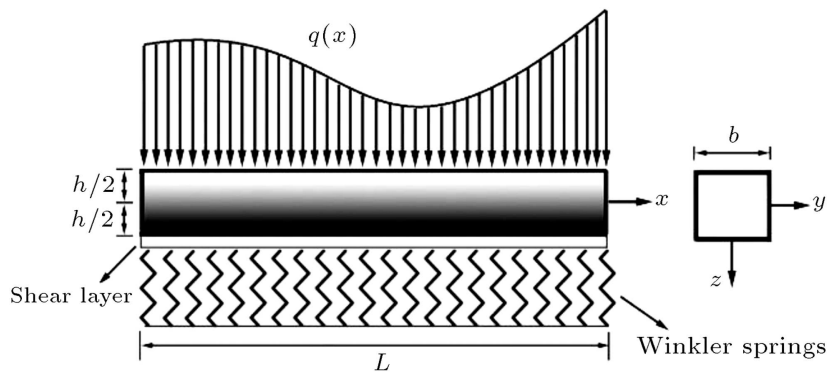

Figure 1. Geometry of a functionally graded size-dependent beam. 
tively. The contribution of normal strain to the displacement field is accounted by $g(z) w_{z}(x, t)$ term. In the present research, $f(z)$ and $g(z)$ functions in Eq. (1) are assumed as follows [60]:

$$
\begin{aligned}
& f(z)=\frac{\frac{h}{\pi} \sinh \left(\frac{\pi}{h} z\right)-z}{\cosh \left(\frac{\pi}{2}\right)-1}, \\
& g(z)=1-\frac{\mathrm{d} f(z)}{\mathrm{d} z} .
\end{aligned}
$$

Based upon the assumed displacement field in Eq. (1), the non-zero strain-displacement terms can be achieved through differentiation respect to the variables $x$ and $z$ below:

$$
\begin{aligned}
& \varepsilon_{x}=\frac{\mathrm{d} u_{0}}{\mathrm{~d} x}-z \frac{\mathrm{d}^{2} w_{b}}{\mathrm{~d} x^{2}}-f(z) \frac{\mathrm{d}^{2} w_{s}}{\mathrm{~d} x^{2}}, \\
& \varepsilon_{z}=\frac{\mathrm{d} g(z)}{\mathrm{d} z} w_{z}, \\
& 2 \varepsilon_{x z}=g(z)\left(\frac{\mathrm{d} w_{s}}{\mathrm{~d} x}+\frac{\mathrm{d} w_{z}}{\mathrm{~d} x}\right) .
\end{aligned}
$$

\subsection{Functionally Graded Materials (FGM)}

\subsubsection{Classical rule of mixture}

The effective material properties of the FG beam using classical rule of mixture are given below:

$$
\begin{aligned}
& E(z)=E_{m}+\left(E_{c}-E_{m}\right)\left(\frac{1}{2}+\frac{z}{h}\right)^{p}, \\
& \nu(z)=\nu_{m}+\left(\nu_{c}-\nu_{m}\right)\left(\frac{1}{2}+\frac{z}{h}\right)^{p},
\end{aligned}
$$

where subscripts $m$ and $c$ represent metal and ceramic constituents, respectively, and $p$ is the gradient power law index in the $z$ direction.

\subsubsection{Mori-Tanaka homogenization scheme}

Based on the Mori-Tanaka homogenization scheme, the effective bulk modulus $\left(K_{e}\right)$ and the effective shear modulus $\left(G_{e}\right)$ of the FG beam are given by Eq. (5) [61] as shown in Box I, where $V$ is the volume fraction of the phase materials. The volume fraction of metal and ceramic constituents of the FG microbeam can be related as follows:

$$
V_{m}+V_{c}=1
$$

where:

$$
\begin{aligned}
& V_{c}(z)=\left(\frac{z}{h}+\frac{1}{2}\right)^{p}, \\
& V_{m}(z)=1-\left(\frac{z}{h}+\frac{1}{2}\right)^{p} .
\end{aligned}
$$

Consequently, according to Mori-Tanaka homogenization scheme, the effective Young's modulus and Poisson's ratio can be expressed below:

$$
\begin{aligned}
& E(z)=\frac{9 K_{e} G_{e}}{3 K_{e}+G_{e}}, \\
& \nu(z)=\frac{3 K_{e}-2 G_{e}}{6 K_{e}+2 G_{e}} .
\end{aligned}
$$

\subsection{Modified Strain Gradient Theory (MSGT)} According to the MSGT presented by Lam et al. [15], the strain energy stored in a linear elastic continuum, including higher order terms, can be expressed as follows:

$$
U=\frac{1}{2} \int_{\Omega}\left(\sigma_{i j} \varepsilon_{i j}+p_{i} \gamma_{i}+\tau_{i j k}^{(1)} \eta_{i j k}^{(1)}+m_{i j}^{s} \chi_{i j}^{s}\right) \mathrm{d} \Omega
$$

where $\varepsilon_{i j}, \gamma_{i}, \eta_{i j k}^{(1)}$, and $\chi_{i j}^{s}$ are strain tensor, dilatation gradient tensor, deviatoric stretch gradient tensor, and symmetric rotation gradient tensor, respectively, where:

$$
\begin{aligned}
& \varepsilon_{i j}=\frac{1}{2}\left(u_{i, j}+u_{j, i}\right), \\
& \gamma_{i}=\varepsilon_{m m, i}, \\
& \eta_{i j k}=\eta_{i j k}^{s}-\frac{1}{5}\left(\delta_{i j} \eta_{m m k}^{s}+\delta_{j k} \eta_{m m i}^{s}+\delta_{j k} \eta_{m m j}^{s}\right), \\
& \chi_{i j}^{s}=\frac{1}{4}\left(e_{i p q} \varepsilon_{q j, p}+e_{j p q} \varepsilon_{q i, p}\right),
\end{aligned}
$$

in which:

$$
\eta_{i j k}^{s}=\frac{1}{3}\left(u_{i, j k}+u_{j, k i}+u_{k, i j}\right) .
$$

According to the assumed displacement field, the nonzero coefficients of Eq. (10) are as follows:

$$
\begin{aligned}
& \varepsilon_{11}=\frac{\mathrm{d} u}{\mathrm{~d} x}-z \frac{\mathrm{d}^{2} w_{b}}{\mathrm{~d} x^{2}}-f \frac{\mathrm{d}^{2} w_{s}}{\mathrm{~d} x^{2}}, \\
& \varepsilon_{13}=\frac{1}{2} g\left(\frac{\mathrm{d} w_{s}}{\mathrm{~d} x}+\frac{\mathrm{d} w_{z}}{\mathrm{~d} x}\right),
\end{aligned}
$$

$$
\begin{aligned}
& \frac{K_{e}-K_{m}}{K_{c}-K_{m}}=\frac{V_{c}}{1+V_{m}\left(K_{c}-K_{m}\right) /\left(K_{m}+4 G_{m} / 3\right)} \\
& \frac{G_{e}-G_{m}}{G_{c}-G_{m}}=\frac{V_{c}}{1+V_{m}\left(G_{c}-G_{m}\right) /\left[G_{m}+G_{m}\left(9 K_{m}+8 G_{m}\right) /\left(6\left(K_{m}+2 G_{m}\right)\right)\right]}
\end{aligned}
$$




$$
\begin{aligned}
& \varepsilon_{33}=\frac{1}{2} \frac{\mathrm{d} g}{\mathrm{~d} z} w_{z} \\
& \gamma_{1}=\frac{\mathrm{d}^{2} u}{\mathrm{~d} x^{2}}-z \frac{\mathrm{d}^{3} w_{b}}{\mathrm{~d} x^{3}}-f \frac{\mathrm{d}^{3} w_{s}}{\mathrm{~d} x^{3}}+\frac{\mathrm{d} g}{\mathrm{~d} z} \frac{\mathrm{d} w_{z}}{\mathrm{~d} x}, \\
& \gamma_{3}=-\frac{\mathrm{d}^{2} w_{b}}{\mathrm{~d} x^{2}}-\frac{\mathrm{d} f}{\mathrm{~d} z} \frac{\mathrm{d}^{2} w_{s}}{\mathrm{~d} x^{2}}+\frac{\mathrm{d}^{2} g}{\mathrm{~d} z^{2}} w_{z}, \\
& \eta_{111}=\frac{2}{5} \frac{\mathrm{d}^{2} u}{\mathrm{~d} x^{2}}-\frac{2}{5} z \frac{\mathrm{d}^{3} w_{b}}{\mathrm{~d} x^{3}}-\frac{2}{5} f \frac{\mathrm{d}^{3} w_{s}}{\mathrm{~d} x^{3}} \\
& -\frac{2}{5} \frac{\mathrm{d} g}{\mathrm{~d} z} \frac{\mathrm{d} w_{z}}{\mathrm{~d} x}+\frac{1}{5} \frac{\mathrm{d}^{2} f}{\mathrm{~d} z^{2}} \frac{\mathrm{d} w_{s}}{\mathrm{~d} x} \\
& \eta_{113}=\eta_{311}=\eta_{131}=-\frac{8}{5} \frac{\mathrm{d} f}{\mathrm{~d} z} \frac{\mathrm{d}^{2} w_{s}}{\mathrm{~d} x^{2}}+\frac{4}{15} \frac{\mathrm{d}^{2} w_{s}}{\mathrm{~d} x^{2}} \\
& +\frac{4}{15} g \frac{\mathrm{d}^{2} w_{z}}{\mathrm{~d} x^{2}}-\frac{4}{15} \frac{\mathrm{d}^{2} w_{b}}{\mathrm{~d} x^{2}}-\frac{1}{5} \frac{\mathrm{d}^{2} g}{\mathrm{~d} z^{2}} w_{z} \\
& \eta_{133}=\eta_{313}=\eta_{331}=\frac{8}{15} \frac{\mathrm{d} g}{\mathrm{~d} z} \frac{\mathrm{d} w_{z}}{\mathrm{~d} x}-\frac{4}{15} \frac{\mathrm{d}^{2} f}{\mathrm{~d} z^{2}} \frac{\mathrm{d} w_{s}}{\mathrm{~d} x} \\
& -\frac{1}{5} \frac{\mathrm{d}^{2} u}{\mathrm{~d} x^{2}}+\frac{1}{5} z \frac{\mathrm{d}^{3} w_{b}}{\mathrm{~d} x^{3}}+\frac{1}{5} f \frac{\mathrm{d}^{3} w_{s}}{\mathrm{~d} x^{3}}, \\
& \eta_{122}=\eta_{212}=\eta_{221}=-\frac{1}{5} \frac{\mathrm{d}^{2} u}{\mathrm{~d} x^{2}}+\frac{1}{5} z \frac{\mathrm{d}^{3} w_{b}}{\mathrm{~d} x^{3}}+\frac{1}{5} f \frac{\mathrm{d}^{3} w_{s}}{\mathrm{~d} x^{3}} \\
& -\frac{2}{5} \frac{\mathrm{d} g}{\mathrm{~d} z} \frac{\mathrm{d} w_{z}}{\mathrm{~d} x}+\frac{1}{15} \frac{\mathrm{d}^{2} f}{\mathrm{~d} z^{2}} \frac{\mathrm{d} w_{s}}{\mathrm{~d} x}, \\
& \eta_{223}=\eta_{322}=\eta_{232}=\frac{1}{15} \frac{\mathrm{d}^{2} w_{b}}{\mathrm{~d} x^{2}}+\frac{2}{15} \frac{\mathrm{d} f}{\mathrm{~d} z} \frac{\mathrm{d}^{2} w_{s}}{\mathrm{~d} x^{2}} \\
& -\frac{1}{5} \frac{\mathrm{d}^{2} g}{\mathrm{~d} z^{2}} w_{z}-\frac{1}{15} \frac{\mathrm{d}^{2} w_{s}}{\mathrm{~d} x^{2}}-\frac{1}{15} g \frac{\mathrm{d}^{2} w_{z}}{\mathrm{~d} x^{2}}, \\
& \eta_{333}=\frac{2}{5} \frac{\mathrm{d}^{2} g}{\mathrm{~d} z^{2}} w_{z}+\frac{1}{5} \frac{\mathrm{d}^{2} w_{b}}{\mathrm{~d} x^{2}}+\frac{2}{5} \frac{\mathrm{d} f}{\mathrm{~d} z} \frac{\mathrm{d}^{2} w_{s}}{\mathrm{~d} x^{2}} \\
& -\frac{1}{5} \frac{\mathrm{d}^{2} w_{s}}{\mathrm{~d} x^{2}}-\frac{1}{5} g \frac{\mathrm{d}^{2} w_{z}}{\mathrm{~d} x^{2}}, \\
& \chi_{i j}^{s}=\frac{1}{4}\left(e_{i p q} \varepsilon_{q j, p}+e_{j p q} \varepsilon_{q i, p}\right) .
\end{aligned}
$$

The corresponding classical stress field associated with the above strain terms is given below [62]:

$$
\left\{\begin{array}{l}
\sigma_{11} \\
\sigma_{33} \\
\sigma_{13}
\end{array}\right\}=\left[\begin{array}{ccc}
\frac{E(z)}{1-\nu^{2}(z)} & \frac{E(z) \cdot \nu(z)}{1-\nu^{2}(z)} & 0 \\
\frac{E(z) \cdot \nu(z)}{1-\nu^{2}(z)} & \frac{E(z)}{1-\nu^{2}(z)} & 0 \\
0 & 0 & \frac{E(z)}{2(1+\nu(z))}
\end{array}\right]\left\{\begin{array}{c}
\varepsilon_{11} \\
\varepsilon_{33} \\
2 \varepsilon_{13}
\end{array}\right\} .
$$

In addition, higher order stresses can be expressed as:

$$
\begin{aligned}
& p_{i}=2 \mu l_{0}^{2} \gamma_{i}, \\
& \tau_{i j k}^{1}=2 \mu l_{1}^{2} \eta_{i j k}, \\
& m_{i j}^{s}=2 \mu l_{2}^{2} \chi_{i j}^{s},
\end{aligned}
$$

where $l_{0}, l_{1}$ and $l_{2}$ are three length scale parameters in MSGT. This theory can be converted to MCST by allowing $l_{0}=l_{1}=0$ and to CBT by $l_{0}=l_{1}=l_{2}=0$.

\subsection{Governing equations}

In order to obtain the governing equations, the minimum total potential energy principle is used as follows:

$$
\delta \int_{V}\left(U+U_{f}-W\right) \mathrm{d} t=0,
$$

where $U$ is the strain energy, $U_{f}$ is the strain energy of elastic foundation, and $W$ is the work done by the external applied forces. The strain energy induced by elastic foundation will be:

$$
\begin{aligned}
U_{f}= & \frac{1}{2} \int_{A} k_{w}\left\{w_{b}{ }^{2}+w_{s}^{2}\right\} \mathrm{d} A \\
& +\frac{1}{2} \int_{A} k_{p}\left\{\frac{\mathrm{d}\left(w_{b}+w_{s}\right)}{\mathrm{d} x}\right\}^{2} \mathrm{~d} A,
\end{aligned}
$$

where $k_{w}$ and $k_{p}$ are Winkler and Pasternak stiffness parameters of the elastic foundation, respectively. The work done by external force can be obtained as follows:

$$
W=\int_{A} q\left\{w_{b}+w_{s}\right\} \mathrm{d} A .
$$

By substituting Eqs. (9), (16), (17) into Eq. (15) through some mathematical processes and employing integration by the technique, the following governing equations and corresponding boundary conditions can be achieved:

$$
\begin{aligned}
\delta u: & -\frac{\mathrm{d} N_{11}}{\mathrm{~d} x}+\frac{\mathrm{d}^{2} P_{1}}{\mathrm{~d} x^{2}}+\frac{2}{5} \frac{\mathrm{d}^{2} T_{111}}{\mathrm{~d} x^{2}}-\frac{3}{5} \frac{\mathrm{d}^{2} T_{133}}{\mathrm{~d} x^{2}} \\
& -\frac{3}{5} \frac{\mathrm{d}^{2} T_{122}}{\mathrm{~d} x^{2}}=0 \\
\delta w_{b}: & -\frac{\mathrm{d}^{2} N_{11}^{a}}{\mathrm{~d} x^{2}}-\frac{\mathrm{d}^{2} M_{12}}{\mathrm{~d} x^{2}}-\frac{\mathrm{d}^{2} P_{1}^{a}}{\mathrm{~d} x^{2}}+\frac{2}{5} \frac{\mathrm{d}^{3} T_{111}^{a}}{\mathrm{~d} x^{3}} \\
& -\frac{4}{5} \frac{\mathrm{d}^{3} T_{113}}{\mathrm{~d} x^{3}}-\frac{3}{5} \frac{\mathrm{d}^{3} T_{133}^{a}}{\mathrm{~d} x^{3}}-\frac{3}{5} \frac{\mathrm{d}^{3} T_{122}^{a}}{\mathrm{~d} x^{3}} \\
& +\frac{1}{5} \frac{\mathrm{d}^{2} T_{223}}{\mathrm{~d} x^{2}}+\frac{1}{5} \frac{\mathrm{d}^{2} T_{333}}{\mathrm{~d} x^{2}}+k_{w}\left(w_{b}+w_{s}\right) \\
& -k_{P}\left(\frac{\mathrm{d}^{2} w_{b}}{\mathrm{~d} x^{2}}+\frac{\mathrm{d}^{2} w_{s}}{\mathrm{~d} x^{2}}\right)-q=0, \\
\delta w_{s}: & -\frac{\mathrm{d}^{2} N_{11}^{b}}{\mathrm{~d} x^{2}}+2 \frac{\mathrm{d} S_{13}}{\mathrm{~d} x}-\frac{\mathrm{d}^{2} M_{12}}{\mathrm{~d} x^{2}}+\frac{1}{2} \frac{\mathrm{d}^{2} M_{12}^{a}}{\mathrm{~d} x^{2}} \\
& -\frac{1}{2} \frac{\mathrm{d} M_{23}}{\mathrm{~d} x}-\frac{\mathrm{d}^{2} P_{1}^{b}}{\mathrm{~d} x^{2}}-\frac{\mathrm{d}^{2} P_{3}^{a}}{\mathrm{~d} x^{2}}+\frac{2}{5} \frac{\mathrm{d}^{3} T_{111}^{b}}{\mathrm{~d} x^{3}} \\
& -\frac{1}{5} \frac{\mathrm{d} T_{111}^{d}}{\mathrm{~d} x}-\frac{8}{5} \frac{\mathrm{d}^{2} T_{113}^{b}}{\mathrm{~d} x^{2}}+\frac{4}{5} \frac{\mathrm{d}^{2} T_{113}}{\mathrm{~d} x^{2}}+\frac{4}{5} \frac{\mathrm{d} T_{133}^{d}}{\mathrm{~d} x}
\end{aligned}
$$




$$
\begin{aligned}
+ & \frac{3}{5} \frac{\mathrm{d} T_{133}^{b}}{\mathrm{~d} x}-\frac{3}{5} \frac{\mathrm{d}^{3} T_{122}}{\mathrm{~d} x^{3}}-\frac{1}{5} \frac{\mathrm{d} T_{122}^{d}}{\mathrm{~d} x}+\frac{2}{5} \frac{\mathrm{d}^{2} T_{223}^{b}}{\mathrm{~d} x^{2}} \\
& -\frac{1}{5} \frac{\mathrm{d}^{2} T_{223}}{\mathrm{~d} x^{2}}+\frac{2}{5} \frac{\mathrm{d}^{2} T_{333}^{b}}{\mathrm{~d} x^{2}}-\frac{1}{5} \frac{\mathrm{d}^{2} T_{333}}{\mathrm{~d} x^{2}} \\
& +k_{w}\left(w_{b}+w_{s}\right)-k_{P}\left(\frac{\mathrm{d}^{2} w_{b}}{\mathrm{~d} x^{2}}+\frac{\mathrm{d}^{2} w_{s}}{\mathrm{~d} x^{2}}\right)-q=0 \\
\delta w_{z}: & -2 \frac{\mathrm{d} S_{13}}{\mathrm{~d} x}+R_{33}-\frac{1}{2} \frac{\mathrm{d}^{2} M_{12}^{a}}{\mathrm{~d} x^{2}}+\frac{1}{2} \frac{\mathrm{d} M_{23}}{\mathrm{~d} x} \\
& -\frac{\mathrm{d} P_{1}^{c}}{\mathrm{~d} x}+P_{3}^{b}+\frac{2}{5} \frac{\mathrm{d} T_{111}^{c}}{\mathrm{~d} x}+\frac{4}{5} \frac{\mathrm{d}^{2} T_{113}^{a}}{\mathrm{~d} x^{2}}-\frac{3}{5} T_{113}^{c} \\
& -\frac{8}{5} \frac{\mathrm{d} T_{133}^{c}}{\mathrm{~d} x}+\frac{2}{5} \frac{\mathrm{d} T_{122}^{c}}{\mathrm{~d} x}-\frac{3}{5} T_{223}^{c}-\frac{1}{5} \frac{\mathrm{d}^{2} T_{223}^{a}}{\mathrm{~d} x^{2}} \\
+ & \frac{2}{5} T_{333}^{c}-\frac{1}{5} \frac{\mathrm{d}^{2} T_{333}^{a}}{\mathrm{~d} x^{2}}=0,
\end{aligned}
$$

where stress resultants in the above equations can be calculated by integrating through the thickness as follows:

$$
\begin{aligned}
& \left\{N_{11}, N_{11}^{a}, N_{11}^{b}\right\}=\int_{-h / 2}^{h / 2}\left\{\sigma_{11}, \sigma_{11} z, \sigma_{11} f\right\} \mathrm{d} A \\
& S_{13}=\int_{-h / 2}^{h / 2} \sigma_{13} g \mathrm{~d} A, \\
& R_{33}=\int_{-h / 2}^{h / 2} \sigma_{33} \frac{\mathrm{d} g}{\mathrm{~d} z} \mathrm{~d} A, \\
& \left\{M_{12}, M_{12}^{a}, M_{23}\right\}=\int_{-h / 2}^{h / 2}\left\{m_{12}, \sigma_{11} g, \sigma_{23}\right\} \mathrm{d} A \\
& \left\{P_{1}, P_{1}^{a}, P_{1}^{b}, P_{1}^{c}\right\}=\int_{-h / 2}^{h / 2}\left\{p_{1}, p_{1} z, p_{1} f, p_{1} \frac{\mathrm{d} g}{\mathrm{~d} z}\right\} \mathrm{d} A \\
& \quad=\int_{-h / 2}^{h / 2}\left\{\tau_{113}, \tau_{113} g, \tau_{113} \frac{\mathrm{d} f}{\mathrm{~d} z}, \tau_{113} \frac{\mathrm{d}^{2} g}{\mathrm{~d} z^{2}}\right\} \mathrm{d} A \\
& \left\{P_{3}, P_{3}^{a}, P_{3}^{b}\right\}=\int_{-h / 2}^{h / 2}\left\{p_{3}, p_{3} \frac{\mathrm{d} f}{\mathrm{~d} z}, p_{3} \frac{\mathrm{d}^{2} g}{\mathrm{~d} z^{2}}\right\} \mathrm{d} A \\
& \left\{T_{111}, T_{111}^{a}, T_{111}^{b}, T_{111}^{c}, T_{111}^{d}\right\} \\
& =\int_{-h / 2}^{h / 2}\left\{\tau_{111}^{b}, \tau_{111} z, \tau_{111} f, \tau_{111} \frac{\mathrm{d} g}{\mathrm{~d} z}, \tau_{111} \frac{\mathrm{d}^{2} f}{\mathrm{~d} z^{2}}\right\} \mathrm{d} A \\
& \left\{T_{113}, T_{113}^{a}, T_{113}^{b}, T_{113}^{c}\right\}
\end{aligned}
$$

$$
\begin{aligned}
& \left\{T_{122}, T_{122}^{a}, T_{122}^{b}, T_{122}^{c}, T_{122}^{d}\right\} \\
& \quad=\int_{-h / 2}^{h / 2}\left\{\tau_{122}, \tau_{122} z, \tau_{122} f, \tau_{122} \frac{\mathrm{d} g}{\mathrm{~d} z}, \tau_{122} \frac{\mathrm{d}^{2} f}{\mathrm{~d} z^{2}}\right\} \mathrm{d} A \\
& \left\{T_{223}, T_{223}^{a}, T_{223}^{b}, T_{223}^{c}\right\} \\
& \quad=\int_{-h / 2}^{h / 2}\left\{\tau_{223}, \tau_{223} g, \tau_{223} \frac{\mathrm{d} f}{\mathrm{~d} z}, \tau_{223} \frac{\mathrm{d}^{2} g}{\mathrm{~d} z^{2}}\right\} \mathrm{d} A, \\
& \left(T_{333}, T_{333}^{a}, T_{333}^{b}, T_{333}^{c}\right) \\
& \quad=\int_{-h / 2}^{h / 2}\left(\tau_{333}, \tau_{333} g, \tau_{333} \frac{\mathrm{d} f}{\mathrm{~d} z}, \tau_{333} \frac{\mathrm{d}^{2} g}{\mathrm{~d} z^{2}}\right) \mathrm{d} A .
\end{aligned}
$$

By substituting Eqs. (12)-(14) into Eq. (19), the components of classical stress resultants in terms of displacements can be obtained as follows:

$$
\begin{aligned}
& \left\{\begin{array}{l}
N_{11} \\
N_{11}^{a} \\
N_{11}^{b}
\end{array}\right\}=\left\{\begin{array}{l}
A_{1} \\
A_{2} \\
A_{3}
\end{array}\right\} \frac{\mathrm{d} u}{\mathrm{~d} x}-\left\{\begin{array}{l}
A_{2} \\
A_{5} \\
A_{4}
\end{array}\right\} \frac{\mathrm{d}^{2} w_{b}}{\mathrm{~d} x^{2}}-\left\{\begin{array}{l}
A_{3} \\
A_{4} \\
A_{6}
\end{array}\right\} \frac{\mathrm{d}^{2} w_{s}}{\mathrm{~d} x^{2}} \\
& +\left\{\begin{array}{l}
B_{1} \\
B_{2} \\
B_{3}
\end{array}\right\} w_{z}, \\
& R_{33}=B_{1} \frac{\mathrm{d} u}{\mathrm{~d} x}-B_{2} \frac{\mathrm{d}^{2} w_{b}}{\mathrm{~d} x^{2}}-B_{3} \frac{\mathrm{d}^{2} w_{s}}{\mathrm{~d} x^{2}}+A_{7} w_{z}, \\
& S_{13}=\frac{1}{2} D_{8}\left(\frac{\mathrm{d} w_{s}}{\mathrm{~d} x}+\frac{\mathrm{d} w_{z}}{\mathrm{~d} x}\right),
\end{aligned}
$$

and the higher order stress resultants can be achieved as follows:

$$
\begin{aligned}
& \left\{\begin{array}{c}
P \\
P_{1}^{a} \\
P_{1}^{b} \\
P_{1}^{c}
\end{array}\right\}=2 l_{0}^{2}\left(\left\{\begin{array}{l}
D_{1} \\
D_{2} \\
D_{3} \\
E_{2}
\end{array}\right\} \frac{\mathrm{d}^{2} u}{\mathrm{~d} x^{2}}-\left\{\begin{array}{c}
D_{2} \\
D_{6} \\
D_{5} \\
E_{3}
\end{array}\right\} \frac{\mathrm{d}^{3} w_{b}}{\mathrm{~d} x^{3}}\right. \\
& \left.-\left\{\begin{array}{l}
D_{3} \\
D_{5} \\
D_{7} \\
E_{4}
\end{array}\right\} \frac{\mathrm{d}^{3} w_{s}}{\mathrm{~d} x^{3}}+\left\{\begin{array}{l}
E_{2} \\
E_{3} \\
E_{4} \\
E_{8}
\end{array}\right\} \frac{\mathrm{d} w_{z}}{\mathrm{~d} x}\right), \\
& \left\{\begin{array}{l}
P_{3} \\
P_{3}^{a} \\
P_{3}^{b}
\end{array}\right\}=2 l_{0}^{2}\left(-\left\{\begin{array}{l}
D_{1} \\
E_{1} \\
F_{2}
\end{array}\right\} \frac{\mathrm{d}^{2} w_{b}}{\mathrm{~d} x^{2}}\right. \\
& \left.-\left\{\begin{array}{l}
E_{1} \\
E_{7} \\
F_{5}
\end{array}\right\} \frac{\mathrm{d}^{2} w_{s}}{\mathrm{~d} x^{2}}+\left\{\begin{array}{l}
F_{2} \\
F_{5} \\
F_{8}
\end{array}\right\} w_{z}\right), \\
& \left\{\begin{array}{l}
M_{12} \\
M_{12}^{a}
\end{array}\right\}=-l_{2}^{2}\left\{\begin{array}{l}
D_{1} \\
D_{4}
\end{array}\right\}\left(\frac{\mathrm{d}^{2} w_{b}}{\mathrm{~d} x^{2}}+\frac{\mathrm{d}^{2} w_{s}}{\mathrm{~d} x^{2}}\right) \\
& +\frac{1}{2} l_{2}^{2}\left\{\begin{array}{l}
D_{4} \\
D_{8}
\end{array}\right\}\left(\frac{\mathrm{d}^{2} w_{s}}{\mathrm{~d} x^{2}}-\frac{\mathrm{d}^{2} w_{z}}{\mathrm{~d} x^{2}}\right),
\end{aligned}
$$




$$
\begin{aligned}
M_{23}=\frac{1}{2} l_{2}^{2} E_{8}\left(\frac{\mathrm{d} w_{s}}{\mathrm{~d} x}-\frac{\mathrm{d} w_{z}}{\mathrm{~d} x}\right) & \left.\begin{array}{l}
T_{111} \\
T_{111}^{a} \\
T_{111}^{b} \\
T_{111}^{c} \\
T_{111}^{d}
\end{array}\right\}=2 l_{1}^{2}\left(\begin{array}{l}
2 \\
5 \\
D_{3} \\
E_{2} \\
F_{1}
\end{array}\right\} \frac{\mathrm{d}^{2} u}{\mathrm{~d} x^{2}}-\frac{2}{5}\left\{\begin{array}{l}
D_{2} \\
D_{6} \\
D_{5} \\
E_{3} \\
F_{3}
\end{array}\right\} \frac{\mathrm{d}^{3} w_{b}}{\mathrm{~d} x^{3}} \\
& -\frac{2}{5}\left\{\begin{array}{l}
D_{3} \\
D_{5} \\
D_{7} \\
E_{4} \\
F_{9}
\end{array}\right\} \frac{\mathrm{d}^{3} w_{s}}{\mathrm{~d} x^{3}}-\frac{2}{5}\left\{\begin{array}{l}
E_{2} \\
E_{3} \\
E_{4} \\
E_{8} \\
F_{6}
\end{array}\right\} \frac{\mathrm{d} w_{z}}{\mathrm{~d} x} \\
+ & \left.\frac{1}{5}\left\{\begin{array}{l}
F_{1} \\
F_{3} \\
F_{9} \\
F_{6} \\
F_{7}
\end{array}\right\} \frac{\mathrm{d} w_{s}}{\mathrm{~d} x}\right),
\end{aligned}
$$$$
\left\{\begin{array}{l}
T_{113} \\
T_{133}^{a} \\
T_{133}^{b} \\
T_{133}^{c} \\
T_{133}^{d}
\end{array}\right\}=2 l_{1}^{2}\left(\frac{8}{15}\left\{\begin{array}{l}
E_{2} \\
E_{3} \\
E_{4} \\
E_{8} \\
F_{6}
\end{array}\right\} \frac{\mathrm{d} w_{z}}{\mathrm{~d} x}-\frac{4}{15}\left\{\begin{array}{l}
F_{1} \\
F_{3} \\
F_{9} \\
F_{6} \\
F_{7}
\end{array}\right\} \frac{\mathrm{d} w_{s}}{\mathrm{~d} x}\right.
$$$$
-\frac{1}{5}\left\{\begin{array}{l}
D_{1} \\
D_{2} \\
D_{3} \\
E_{2} \\
F_{1}
\end{array}\right\} \frac{\mathrm{d}^{2} u}{\mathrm{~d} x^{2}}+\frac{1}{5}\left\{\begin{array}{l}
D_{2} \\
D_{6} \\
D_{5} \\
E_{3} \\
F_{3}
\end{array}\right\} \frac{\mathrm{d}^{3} w_{b}}{\mathrm{~d} x^{3}}
$$$$
\left.+\frac{1}{5}\left\{\begin{array}{l}
D_{3} \\
D_{5} \\
D_{7} \\
E_{4} \\
F_{9}
\end{array}\right\} \frac{\mathrm{d}^{3} w_{s}}{\mathrm{~d} x^{3}}\right)
$$$$
\left\{\begin{array}{l}
T_{223} \\
T_{223}^{a} \\
T_{223}^{b} \\
T_{223}^{c}
\end{array}\right\}=2 l_{1}^{2}\left(\frac{1}{15}\left\{\begin{array}{l}
D_{1} \\
D_{4} \\
E_{1} \\
F_{2}
\end{array}\right\} \frac{\mathrm{d}^{2} w_{b}}{\mathrm{~d} x^{2}}+\frac{2}{15}\left\{\begin{array}{l}
E_{1} \\
E_{5} \\
E_{7} \\
F_{5}
\end{array}\right\} \frac{\mathrm{d}^{2} w_{s}}{\mathrm{~d} x^{2}}\right.
$$$$
\left\{\begin{array}{l}
T_{113} \\
T_{113}^{a} \\
T_{113}^{b} \\
T_{113}^{c}
\end{array}\right\}=2 l_{1}^{2}\left(-\frac{8}{15}\left\{\begin{array}{l}
E_{1} \\
E_{5} \\
E_{7} \\
F_{5}
\end{array}\right\} \frac{\mathrm{d}^{2} w_{s}}{\mathrm{~d} x^{2}}\right.
$$$$
+\frac{4}{15}\left\{\begin{array}{l}
D_{1} \\
D_{4} \\
E_{1} \\
F_{2}
\end{array}\right\} \frac{\mathrm{d}^{2} w_{s}}{\mathrm{~d} x^{2}}+\frac{4}{15}\left\{\begin{array}{l}
D_{4} \\
D_{8} \\
E_{5} \\
F_{4}
\end{array}\right\} \frac{\mathrm{d}^{2} w_{z}}{\mathrm{~d} x^{2}}
$$$$
\left.-\frac{4}{15}\left\{\begin{array}{l}
D_{1} \\
D_{4} \\
E_{1} \\
F_{2}
\end{array}\right\} \frac{\mathrm{d}^{2} w_{b}}{\mathrm{~d} x^{2}}-\frac{1}{5}\left\{\begin{array}{l}
F_{2} \\
F_{4} \\
F_{5} \\
F_{8}
\end{array}\right\} w_{z}\right)
$$$$
-\frac{1}{5}\left\{\begin{array}{l}
F_{2} \\
F_{4} \\
F_{5} \\
F_{8}
\end{array}\right\} w_{z}-\frac{1}{15}\left\{\begin{array}{l}
D_{1} \\
D_{4} \\
E_{1} \\
F_{2}
\end{array}\right\} \frac{\mathrm{d}^{2} w_{s}}{\mathrm{~d} x^{2}}
$$$$
\left.-\frac{1}{15}\left\{\begin{array}{l}
D_{4} \\
D_{8} \\
E_{5} \\
F_{4}
\end{array}\right\} \frac{\mathrm{d}^{2} w_{z}}{\mathrm{~d} x^{2}}\right)
$$$$
\left\{\begin{array}{l}
T_{333} \\
T_{333}^{a} \\
T_{333}^{b} \\
T_{333}^{c}
\end{array}\right\}=2 l_{1}^{2}\left(\frac{2}{5}\left\{\begin{array}{l}
F_{2} \\
F_{4} \\
F_{5} \\
F_{8}
\end{array}\right\} w_{z}+\frac{1}{5}\left\{\begin{array}{l}
D_{1} \\
D_{4} \\
E_{1} \\
F_{2}
\end{array}\right\} \frac{\mathrm{d}^{2} w_{b}}{\mathrm{~d} x^{2}}\right.
$$$$
\left\{\begin{array}{l}
T_{122} \\
T_{122}^{a} \\
T_{122}^{b} \\
T_{122}^{c} \\
T_{122}^{d}
\end{array}\right\}=2 l_{1}^{2}\left(-\frac{1}{5}\left\{\begin{array}{l}
D_{1} \\
D_{2} \\
D_{3} \\
E_{2} \\
F_{1}
\end{array}\right\} \frac{\mathrm{d}^{2} u}{\mathrm{~d} x^{2}}+\frac{1}{5}\left\{\begin{array}{l}
D_{2} \\
D_{6} \\
D_{5} \\
E_{3} \\
F_{3}
\end{array}\right\} \frac{\mathrm{d}^{3} w_{b}}{\mathrm{~d} x^{3}}\right.
$$$$
+\frac{2}{5}\left\{\begin{array}{l}
E_{1} \\
E_{5} \\
E_{7} \\
F_{5}
\end{array}\right\}_{17} \frac{\mathrm{d}^{2} w_{s}}{\mathrm{~d} x^{2}}-\frac{1}{5}\left\{\begin{array}{l}
D_{1} \\
D_{4} \\
E_{1} \\
F_{2}
\end{array}\right\} \frac{\mathrm{d}^{2} w_{s}}{\mathrm{~d} x^{2}}
$$$$
+\frac{1}{5}\left\{\begin{array}{l}
D_{3} \\
D_{5} \\
D_{7} \\
E_{4} \\
F_{9}
\end{array}\right\} \frac{\mathrm{d}^{3} w_{s}}{\mathrm{~d} x^{3}}-\frac{2}{15}\left\{\begin{array}{l}
E_{2} \\
E_{3} \\
E_{4} \\
E_{8} \\
F_{6}
\end{array}\right\} \frac{\mathrm{d} w_{z}}{\mathrm{~d} x}
$$$$
\left.-\frac{1}{5}\left\{\begin{array}{l}
D_{4} \\
D_{8} \\
E_{5} \\
F_{4}
\end{array}\right\} \frac{\mathrm{d}^{2} w_{z}}{\mathrm{~d} x^{2}}\right)
$$

where:

$$
\left.+\frac{1}{15}\left\{\begin{array}{l}
F_{1} \\
F_{3} \\
F_{9} \\
F_{6} \\
F_{7}
\end{array}\right\} \frac{\mathrm{d} w_{s}}{\mathrm{~d} x}\right)
$$

$$
\begin{aligned}
& \left\{A_{1}, A_{2}, A_{3}, A_{4}, A_{5}, A_{6}, A_{7}\right\} \\
& \quad=\int_{A} \frac{E(z)}{1-\nu^{2}(z)}\left\{1, z, f, z f, z^{2}, f^{2},\left(\frac{\mathrm{d} g}{\mathrm{~d} z}\right)^{2}\right\} \mathrm{d} A,
\end{aligned}
$$




$$
\begin{aligned}
& \left\{B_{1}, B_{2}, B_{3}\right\} \\
& =\int_{A} \frac{E(z) \nu(z)}{1-\nu^{2}(z)}\left\{\frac{\mathrm{d} g}{\mathrm{~d} z}, z \frac{\mathrm{d} g}{\mathrm{~d} z}, f \frac{\mathrm{d} g}{\mathrm{~d} z}\right\} \mathrm{d} A, \\
& \left\{C_{1}, C_{2}, C_{3}, C_{4}, C_{5}, C_{6}\right\} \\
& =\int_{A} \frac{E(z) \nu^{2}(z)}{1-\nu^{2}(z)}\left\{1, g, g^{2}, \frac{\mathrm{d} f}{\mathrm{~d} z}, g \frac{\mathrm{d} f}{\mathrm{~d} z},\left(\frac{\mathrm{d} f}{\mathrm{~d} z}\right)^{2}\right\} \mathrm{d} A, \\
& \left\{D_{1}, D_{2}, D_{3}, D_{4}, D_{5}, D_{6}, D_{7}, D_{8}\right\} \\
& =\int_{A} \mu\left\{1, z, f, g, z f, z^{2}, f^{2}, g^{2}\right\} \mathrm{d} A, \\
& \left\{E_{1}, E_{2}, E_{3}, E_{4}, E_{5}, E_{6}, E_{7}, E_{8}\right\} \\
& =\int_{A} \mu\left\{\frac{\mathrm{d} f}{\mathrm{~d} z}, \frac{\mathrm{d} g}{\mathrm{~d} z}, z \frac{\mathrm{d} g}{\mathrm{~d} z}, f \frac{\mathrm{d} g}{\mathrm{~d} z}, g \frac{\mathrm{d} f}{\mathrm{~d} z},\right. \\
& \left.f g \frac{\mathrm{d} g}{\mathrm{~d} z},\left(\frac{\mathrm{d} f}{\mathrm{~d} z}\right)^{2},\left(\frac{\mathrm{d} g}{\mathrm{~d} z}\right)^{2}\right\} \mathrm{d} A, \\
& \left\{F_{1}, F_{2}, F_{3}, F_{4}, F_{5}, F_{6}, F_{7}, F_{8}, F_{9}\right\}= \\
& \int_{A} \mu\left\{\frac{\mathrm{d}^{2} f}{\mathrm{~d} z^{2}}, \frac{\mathrm{d}^{2} g}{\mathrm{~d} z^{2}}, z \frac{\mathrm{d}^{2} f}{\mathrm{~d} z^{2}}, g \frac{\mathrm{d}^{2} g}{\mathrm{~d} z^{2}}, \frac{\mathrm{d} f}{\mathrm{~d} z} \frac{\mathrm{d}^{2} g}{\mathrm{~d} z^{2}}, \frac{\mathrm{d} g}{\mathrm{~d} z} \frac{\mathrm{d}^{2} f}{\mathrm{~d} z^{2}},\right. \\
& \left.\left(\frac{\mathrm{d}^{2} f}{\mathrm{~d} z^{2}}\right)^{2},\left(\frac{\mathrm{d}^{2} g}{\mathrm{~d} z^{2}}\right)^{2}, f\left(\frac{\mathrm{d}^{2} f}{\mathrm{~d} z^{2}}\right)\right\} \mathrm{d} A,
\end{aligned}
$$

and corresponding boundary conditions at the beam ends $(x=0, L)$ are expressed as:

$$
\begin{aligned}
& \delta u=0 \quad \text { or } \\
& N_{11}-\frac{\mathrm{d} P_{1}}{\mathrm{~d} x}-\frac{2}{5} \frac{\mathrm{d} T_{111}}{\mathrm{~d} x}+\frac{3}{5} \frac{\mathrm{d} T_{133}}{\mathrm{~d} x}+\frac{3}{5} \frac{\mathrm{d} T_{122}}{\mathrm{~d} x}=0, \\
& \delta\left(\frac{\mathrm{d} u}{\mathrm{~d} x}\right)=0 \text { or } \\
& P_{1}+\frac{2}{5} T_{111}-\frac{3}{5} T_{133}-\frac{3}{5} T_{122}=0 \\
& \delta w_{b}=0 \quad \text { or } \\
& \frac{\mathrm{d} N_{11}^{a}}{\mathrm{~d} x}+\frac{\mathrm{d} M_{12}}{\mathrm{~d} x}-\frac{2}{5} \frac{\mathrm{d}^{2} T_{111}^{a}}{\mathrm{~d} x^{2}}+\frac{4}{5} \frac{\mathrm{d} T_{113}}{\mathrm{~d} x}+\frac{3}{5} \frac{\mathrm{d}^{2} T_{133}^{a}}{\mathrm{~d} x^{2}} \\
& \quad+\frac{3}{5} \frac{\mathrm{d}^{2} T_{122}^{a}}{\mathrm{~d} x^{2}}-\frac{1}{5} \frac{\mathrm{d} T_{223}}{\mathrm{~d} x}-\frac{1}{5} \frac{\mathrm{d} T_{333}}{\mathrm{~d} x}-\frac{\mathrm{d}^{2} P_{1}^{a}}{\mathrm{~d} x^{2}}=0,(22 \mathrm{c} \\
& \delta\left(\frac{\mathrm{d} w_{b}}{\mathrm{~d} x}\right)=0 \quad \text { or }
\end{aligned}
$$

$$
\delta w_{z}=0 \quad \text { or }
$$$$
\begin{aligned}
-2 S_{13} & +\frac{1}{2} \frac{\mathrm{d} M_{12}^{a}}{\mathrm{~d} x}-\frac{1}{2} M_{23}+P_{1}^{c}-\frac{2}{5} T_{111}^{c}-\frac{4}{5} \frac{\mathrm{d} T_{113}^{a}}{\mathrm{~d} x} \\
& +\frac{8}{5} T_{133}^{c}-\frac{2}{5} T_{122}^{c}+\frac{1}{5} \frac{\mathrm{d} T_{223}^{a}}{\mathrm{~d} x}+\frac{1}{5} \frac{\mathrm{d} T_{333}^{a}}{\mathrm{~d} x}=0
\end{aligned}
$$

$$
\delta\left(\frac{\mathrm{d} w_{z}}{\mathrm{~d} x}\right)=0 \quad \text { or }
$$$$
-\frac{1}{2} M_{12}^{a}+\frac{4}{5} T_{113}^{a}-\frac{1}{5} T_{223}^{a}-\frac{1}{5} T_{333}^{a}=0 .
$$ 


\section{Solution methodology}

In this section, the Navier exact closed-form solution is employed in order to automatically satisfy the simply supported boundary conditions. In the latter method, the displacement terms are expressed as functions with undetermined coefficients. The beam is supposed to be simply supported at $x=0, L$. The expansion of displacement field is expressed as:

$$
\begin{aligned}
& u(x)=\sum_{m=1}^{N} u_{m} \cos \left(\frac{m \pi}{L} x\right), \\
& w_{b}(x)=\sum_{m=1}^{N} w_{b m} \sin \left(\frac{m \pi}{L} x\right), \\
& w_{s}(x)=\sum_{m=1}^{N} w_{s m} \sin \left(\frac{m \pi}{L} x\right), \\
& w_{z}(x)=\sum_{m=1}^{N} w_{z m} \sin \left(\frac{m \pi}{L} x\right),
\end{aligned}
$$

where $u_{m}, w_{b m}, w_{s m}$, and $w_{z m}$ are arbitrary parameters to be determined. In addition, the transverse applied load can be expanded in Fourier series as follows:

$$
q(x)=\sum_{n=1}^{N} Q_{n} \sin \left(\frac{n \pi}{L} x\right),
$$

where:

For single sine load:

$$
Q_{n}=q_{0}, \quad n=1 \text {. }
$$

For uniformly distributed load:

$$
Q_{n}=\frac{4 q_{0}}{n \pi}, \quad n=1,3,5, \ldots
$$

For point load at the middle:

$$
Q_{n}=\frac{2 P}{L} \sin \frac{n \pi x}{L}, \quad n=1,2,3, \ldots
$$

\section{Results and discussion}

In order to demonstrate the accuracy of the present formulation, results for bending of micro beams in a special case $\left(l_{0}=l_{1}=0\right)$ are compared to those in [62]. The FG beam is composed of Sic/Al and associated material properties are expressed in Eq. (26). The classical rule of mixture and Mori-Tanaka scheme are employed to estimate material properties through the thickness. The material length scale parameter in the present study is considered to be $l=15 \mu \mathrm{m}$ [63]. Table 1 shows the comparison of results at different aspect ratios and dimensionless size-dependent parameters for a different power indexes. It can be observed that there is a good agreement between results for both classical rule of mixture and Mori-Tanaka scheme.

$$
\begin{array}{lll}
E_{C} & =427 \mathrm{GPa} & \nu_{C}=0.17, \\
E_{m}=70 \mathrm{GPa} & \nu_{m}=0.3 .
\end{array}
$$

\begin{tabular}{|c|c|c|c|c|c|c|c|c|c|c|}
\hline \multirow[t]{2}{*}{$L / h$} & \multirow[t]{2}{*}{$h / l$} & \multirow[t]{2}{*}{ Theory } & \multicolumn{4}{|c|}{$\bar{w}$ (classical rule of mixture) } & \multicolumn{4}{|c|}{$\overline{\boldsymbol{w}}$ (Mori-Tanaka scheme) } \\
\hline & & & $p=0$ & $p=0.5$ & $p=1$ & $p=10$ & $p=0$ & $p=0.5$ & $p=1$ & $p=10$ \\
\hline \multirow{6}{*}{5} & \multirow{2}{*}{1} & Ref. [62] & 0.0364 & 0.0527 & 0.0663 & 0.1569 & 0.0364 & 0.0713 & 0.0920 & 0.1870 \\
\hline & & Present MCST & 0.0363 & 0.0525 & 0.0660 & 0.1550 & 0.0363 & 0.0708 & 0.0914 & 0.1845 \\
\hline & \multirow{2}{*}{2} & Ref. [62] & 0.0989 & 0.1461 & 0.1861 & 0.4034 & 0.0989 & 0.1975 & 0.2535 & 0.4771 \\
\hline & & Present MCST & 0.0986 & 0.1454 & 0.1851 & 0.3980 & 0.0986 & 0.1964 & 0.2518 & 0.4707 \\
\hline & \multirow[t]{2}{*}{$\infty$} & Ref. [62] & 0.2313 & 0.3567 & 0.4670 & 0.8599 & 0.2313 & 0.4823 & 0.6109 & 0.9956 \\
\hline & & Present CBT & 0.2310 & 0.3550 & 0.4647 & 0.8528 & 0.2306 & 0.4800 & 0.6079 & 0.9876 \\
\hline \multirow{6}{*}{10} & \multirow{2}{*}{1} & Ref. [62] & 0.0352 & 0.0510 & 0.0643 & 0.1522 & 0.0352 & 0.0691 & 0.0894 & 0.1809 \\
\hline & & Present MCST & 0.0352 & 0.0510 & 0.0643 & 0.1518 & 0.0352 & 0.0690 & 0.0893 & 0.1803 \\
\hline & \multirow{2}{*}{2} & Ref. [62] & 0.0949 & 0.1404 & 0.1792 & 0.3838 & 0.0949 & 0.1900 & 0.2437 & 0.4535 \\
\hline & & Present MCST & 0.0949 & 0.1403 & 0.1791 & 0.3824 & 0.0949 & 0.1898 & 0.2434 & 0.4517 \\
\hline & \multirow{2}{*}{$\infty$} & Ref. [62] & 0.2178 & 0.3380 & 0.4429 & 0.7818 & 0.2178 & 0.4554 & 0.5737 & 0.9129 \\
\hline & & Present CBT & 0.2179 & 0.3379 & 0.4426 & 0.7789 & 0.2179 & 0.4552 & 0.5732 & 0.9096 \\
\hline
\end{tabular}

The dimensionless transverse deflections are calculated as follows:

$$
\begin{aligned}
& \bar{w}=\frac{100 E_{m} h^{3}}{12 q_{0} L^{4}} w(x, 0), \quad \hat{w}=\frac{100 E_{m} h^{3}}{12 P L^{3}} w(x, 0), \\
& \bar{\sigma}_{x x}=\frac{\sigma_{x x} h}{q_{0} L}(x, z), \quad \bar{\sigma}_{x z}=\frac{\sigma_{x z} h}{q_{0} L}(x, z), \\
& \bar{k}_{w}=\frac{k_{w} L^{4}}{E I}, \quad \bar{k}_{p}=\frac{k_{p} L^{2}}{E I} .
\end{aligned}
$$

Table 1. Dimensionless deflection of functionally graded micro beam under uniformly distributed loading for different gradient power indexes $\left(q_{0}=10 \mathrm{~N} / \mathrm{m}^{2}\right)$. 
Table 2. Dimensionless deflections of functionally graded size dependent beam under point load: $P=100 \mu$ N.

\begin{tabular}{|c|c|c|c|c|c|c|c|c|c|c|c|c|c|}
\hline \multirow[b]{3}{*}{ Theory } & \multirow[b]{3}{*}{$h / l$} & \multicolumn{6}{|c|}{$\hat{w}$ (classical rule of mixture) } & \multicolumn{6}{|c|}{$\hat{\boldsymbol{w}}$ (Mori-Tanaka scheme) } \\
\hline & & \multicolumn{3}{|c|}{$L / h=10$} & \multicolumn{3}{|c|}{$L / h=100$} & \multicolumn{3}{|c|}{$L / h=10$} & \multicolumn{3}{|c|}{$L / h=100$} \\
\hline & & $p=0$ & $p=1$ & $p=10$ & $p=0$ & $p=1$ & $p=10$ & $p=0$ & $p=1$ & $p=10$ & $p=0$ & $p=1$ & $p=10$ \\
\hline \multirow{4}{*}{$\operatorname{MCST}^{a}$} & 1 & 0.0564 & 0.1029 & 0.2429 & 0.0557 & 0.1017 & 0.2409 & 0.0564 & 0.1429 & 0.2885 & 0.0557 & 0.1416 & 0.2859 \\
\hline & 2 & 0.1520 & 0.2868 & 0.6125 & 0.1497 & 0.2829 & 0.6025 & 0.1520 & 0.3898 & 0.7236 & 0.1497 & 0.3844 & 0.7115 \\
\hline & 4 & 0.2639 & 0.5186 & 0.9919 & 0.2586 & 0.5097 & 0.9643 & 0.2639 & 0.6865 & 1.1641 & 0.2586 & 0.6731 & 1.1332 \\
\hline & 8 & 0.3236 & 0.6502 & 1.1757 & 0.3162 & 0.6375 & 1.1347 & 0.3236 & 0.8481 & 1.3746 & 0.3162 & 0.8286 & 1.3304 \\
\hline \multirow{4}{*}{$\mathrm{MSGT}^{\mathrm{b}}$} & 1 & 0.0348 & 0.0623 & 0.1512 & 0.0346 & 0.0627 & 0.1525 & 0.0348 & 0.0871 & 0.1797 & 0.0346 & 0.0878 & 0.1812 \\
\hline & 2 & 0.1087 & 0.1995 & 0.4462 & 0.1079 & 0.2010 & 0.4494 & 0.1087 & 0.2744 & 0.5276 & 0.1079 & 0.2764 & 0.5313 \\
\hline & 4 & 0.2290 & 0.4370 & 0.8579 & 0.2259 & 0.4399 & 0.8626 & 0.2290 & 0.5831 & 1.0073 & 0.2259 & 0.5865 & 1.0138 \\
\hline & 8 & 0.3112 & 0.5971 & 1.0795 & 0.3050 & 0.6123 & 1.1025 & 0.3112 & 0.7846 & 1.2235 & 0.3050 & 0.7985 & 1.2922 \\
\hline
\end{tabular}

${ }^{a}$ MCST: Modified Couple Stress Theory; ${ }^{b}$ MSGT: Modified Strain Gradient Theory.

Table 3. Effects of foundation parameters on bending of functionally graded size-dependent beam under point load: $P=100 \mu \mathrm{N}, h / l=1$, and $L=50 \mathrm{~h}$.

\begin{tabular}{|c|c|c|c|c|c|c|c|c|c|c|}
\hline \multirow[b]{3}{*}{ Theory } & \multirow[b]{3}{*}{$\bar{k}_{w}$} & \multicolumn{9}{|c|}{$\hat{\boldsymbol{w}}$} \\
\hline & & \multicolumn{3}{|c|}{$p=0$} & \multicolumn{3}{|c|}{$p=1$} & \multicolumn{3}{|c|}{$p=10$} \\
\hline & & $\bar{k}_{p}=0$ & $\overline{\boldsymbol{k}}_{p}=\mathbf{1 0}$ & $\bar{k}_{p}=10^{2}$ & $\bar{k}_{p}=\mathbf{0}$ & $\overline{\boldsymbol{k}}_{p}=\mathbf{1 0}$ & $\bar{k}_{p}=10^{2}$ & $\bar{k}_{p}=\mathbf{0}$ & $\overline{\boldsymbol{k}}_{p}=\mathbf{1 0}$ & $\bar{k}_{p}=10^{2}$ \\
\hline \multirow{4}{*}{$\mathrm{CBT}^{\mathrm{a}}$} & 0 & 0.3418 & 0.2937 & 0.1308 & 0.6960 & 0.5223 & 0.1641 & 1.2072 & 0.7663 & 0.1846 \\
\hline & 10 & 0.3362 & 0.2896 & 0.1300 & 0.6733 & 0.5095 & 0.1629 & 1.1404 & 0.7392 & 0.1831 \\
\hline & $10^{2}$ & 0.2932 & 0.2573 & 0.1233 & 0.5209 & 0.4177 & 0.1527 & 0.7635 & 0.5619 & 0.1707 \\
\hline & $10^{3}$ & 0.1304 & 0.1230 & 0.0817 & 0.1647 & 0.1534 & 0.0953 & 0.1880 & 0.1738 & 0.1038 \\
\hline \multirow{4}{*}{$\mathrm{MCST}^{\mathrm{b}}$} & 0 & 0.0558 & 0.0543 & 0.0440 & 0.1018 & 0.0971 & 0.0685 & 0.2410 & 0.2160 & 0.1125 \\
\hline & 10 & 0.0556 & 0.0542 & 0.0439 & 0.1013 & 0.0966 & 0.0683 & 0.2382 & 0.2138 & 0.1119 \\
\hline & $10^{2}$ & 0.0543 & 0.0529 & 0.0431 & 0.0970 & 0.0927 & 0.0663 & 0.2158 & 0.1956 & 0.1068 \\
\hline & $10^{3}$ & 0.0439 & 0.0430 & 0.0363 & 0.0683 & 0.0661 & 0.0516 & 0.1120 & 0.1064 & 0.0738 \\
\hline \multirow{4}{*}{$\mathrm{MSGT}^{\mathrm{c}}$} & 0 & 0.0347 & 0.0341 & 0.0297 & 0.0628 & 0.0609 & 0.0483 & 0.1524 & 0.1420 & 0.0883 \\
\hline & 10 & 0.0346 & 0.0340 & 0.0297 & 0.0626 & 0.0607 & 0.0481 & 0.1513 & 0.1411 & 0.0879 \\
\hline & $10^{2}$ & 0.0341 & 0.0335 & 0.0293 & 0.0609 & 0.0592 & 0.0472 & 0.1419 & 0.1329 & 0.0847 \\
\hline & $10^{3}$ & 0.0297 & 0.0292 & 0.0260 & 0.0481 & 0.0471 & 0.0392 & 0.0880 & 0.0845 & 0.0623 \\
\hline
\end{tabular}

${ }^{a}$ CBT: Classical Beam Theory; ${ }^{\mathrm{b}} \mathrm{MCST}$ : Modified Couple Stress Theory; ${ }^{\mathrm{c}} \mathrm{MSGT}$ : Modified Strain Gradient Theory.

Table 2 lists dimensionless deflections of MSGT and MCST theories for different aspect ratios $(L / h)$, dimensionless size-dependent parameters $(h / l)$, and power index numbers. The dimensionless deflections for both MSGT and MCST theories increase with the increase of power index numbers and dimensionless size-dependent parameters $(h / l)$. For simplicity and in order to compare the results of different theories, it was assumed that $\left(l_{0}=l_{1}=l_{2}=l\right)$ for MSGT.

Table 3 contains effects of foundation parameters and power law indexes on dimensionless deflection for different theories under point load in the middle of the beam based on the classical rule of mixture. Remarkable variations have been observed for results of CBT, whereas results of MSGT have experienced relatively few changes. Moreover, the effects of dimensionless Pasternak shear parameter $\left(\bar{k}_{p}\right)$ have been more noticeable than dimensionless Winkler parameter $\left(\bar{k}_{w}\right)$.

Figure 2 illustrates the dimensionless deflections for different theories. Results of MSGT and MCST theories are closer to each other than to the results of CBT. Classical continuum theory is not capable of approximating size-dependent behavior and assumes the beam under study to be more flexible (less stiff) than higher order continuum theories (MSGT, MCST). For different theories (CBT, MSGT, MCST), MoriTanaka scheme offers higher values than the classical rule of mixture for dimensionless deflections. 


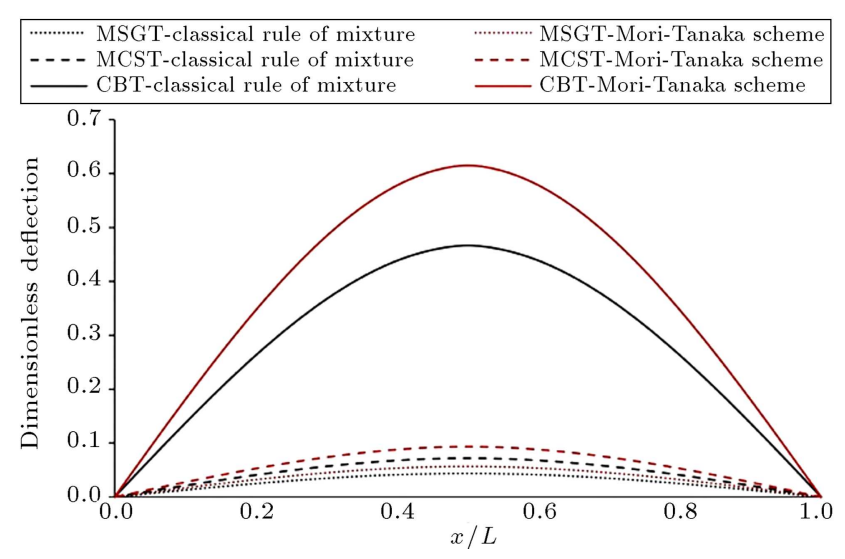

Figure 2. Comparison of the dimensionless static deflection for simply supported micro beam subjected to point load: $P=100 \mu \mathrm{N}(L=10 \mathrm{~h}, p=0.3)$.

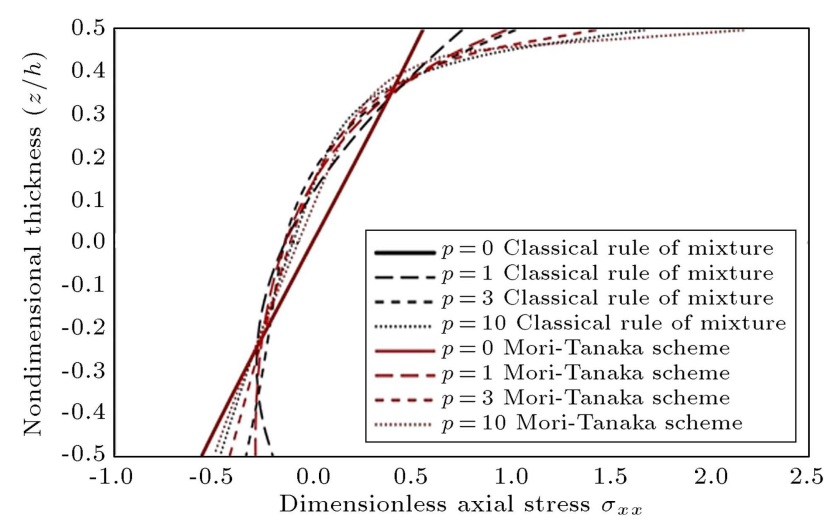

Figure 3. Variation in dimensionless axial stress $\sigma_{x x}$ through non-dimensional thickness for different power index parameters under transverse single sine load $(x=L / 2)$.

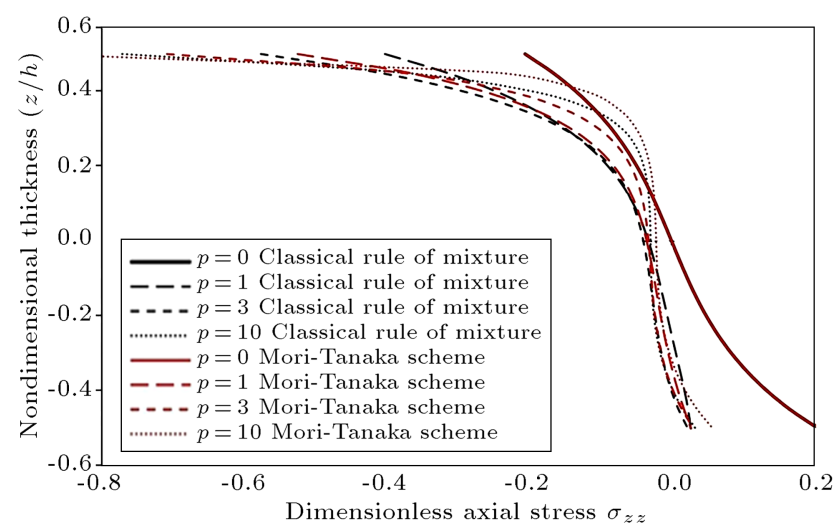

Figure 4. Variation in dimensionless normal stress $\sigma_{z z}$ through non-dimensional thickness for different power index parameters under transverse single sine load $P=100 \mu \mathrm{N}(x=L / 2)$.

Figures 3 and 4 show the changes in dimensionless axial and normal stresses through dimensionless thickness ( $L=10 \mathrm{~h}$ ) based on MSGT theory and under single sine load $q_{0}=10 \mathrm{~N} / \mathrm{m}^{2}$ for different power indexes, respectively. An increase in power index

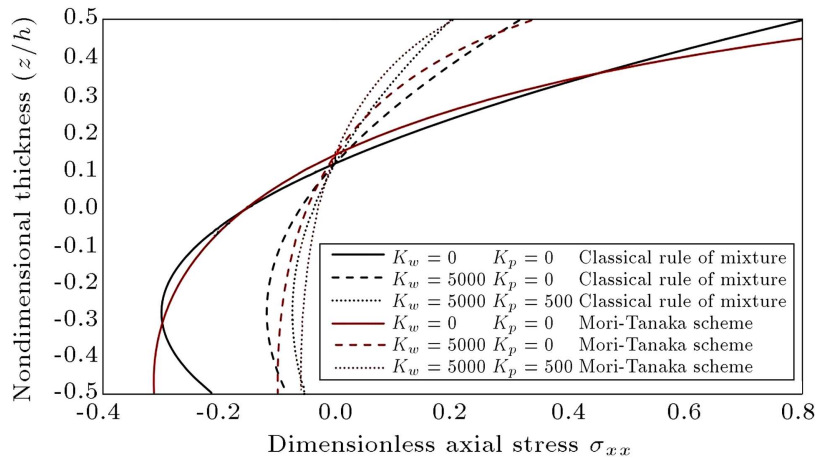

Figure 5. Variation in dimensionless axial stress $\sigma_{x x}$ through non-dimensional thickness for different elastic foundation parameters under transverse single sine load $(x=L / 2)$.

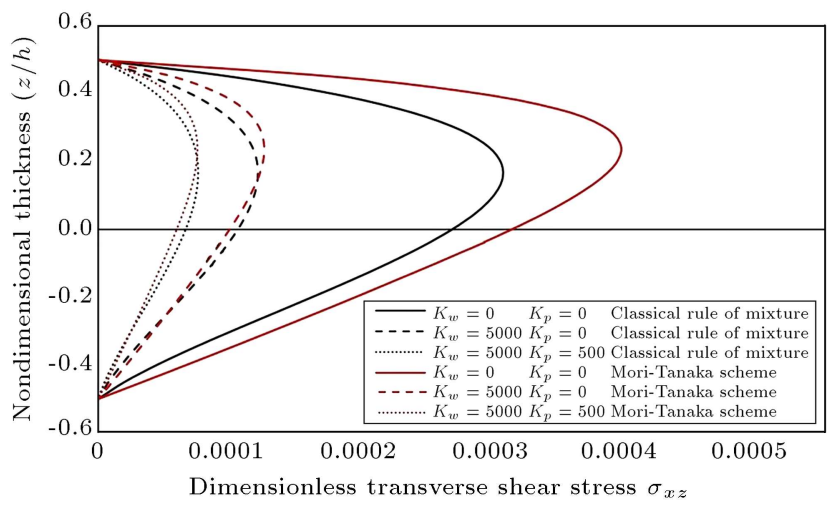

Figure 6. Variation in dimensionless transverse shear stress $\sigma_{x z}$ through non-dimensional thickness for different elastic foundation parameters under transverse single sine load $(x=L / 4)$.

number forces the material constituents to change from ceramic to metal and both axial and normal stresses undergo more obvious changes through nondimensional thickness. For the present dimensionless thickness $(L=10 \mathrm{~h})$, that thickness is comparable to the length of size-dependent beam and the amount of changes for normal stresses are more pronounced than axial stresses. The material properties of the size-dependent FG beams are very important in the bending response, especially in higher gradient indexes $(p=10)$, and the number of variations from bottom to top surface is remarkable.

Figures 5 and 6 depict the variation in nondimensional axial and transverse shear stresses through dimensionless thickness based on MSGT theory for $p=$ 1 and $L=10 \mathrm{~h}$ under single sine load $q_{0}=10 \mathrm{~N} / \mathrm{m}^{2}$, respectively. Effects of foundation parameters were investigated for both dimensionless axial and transverse shear stresses through non-dimensional thickness of the FG size-dependent beams.

In Figures 5 and 6 , inclusion of foundation parameters results in reducing the variation in nondimensional axial and transverse shear stresses through 
dimensionless thickness, respectively. In addition, the calculated dimensionless axial, normal, and transverse shear stresses using Mori-Tanaka scheme follow the same trends as the classical rule of mixture, albeit mostly with slightly higher values. Pasternak parameters (shear layer) resulted in reduction of the dimensionless axial stresses in magnitude compared to Winker elastic foundation and the situation that the elastic foundation is neglected. Results of dimensionless transverse shear stresses (Figure 6) approach are close to one another for Mori-Tanaka and classical rule of mixture schemes as the values of the foundation parameters $\left(K_{w}, K_{p}\right)$ increase.

\section{Conclusion}

In the present study, static bending of a functionally graded size-dependent beam was performed using Modified Strain Gradient Theory (MSGT). Different types of loading, volume fraction indexes, material properties schemes and elastic foundation parameters were investigated for simply supported beams. Stretching effects were also considered in displacement field, which were noticeable in the case of thick beams. Parametric study demonstrated that the impact of the Pasternak coefficient was more significant than the Winkler one for bending behavior. Results of the present paper were compared to the existing ones in special cases (MCST and CBT). It can be seen that the beam behaves stiffer in MSGT than Modified Couple Stress Theory (MCST) theory, because the additional higher order terms were considered. The MCST only involved the rotation gradients as higher order terms in governing equations, while Classical Beam Theory (CBT) was not capable of predicting small-scale behavior. The obtained results illustrated that the inclusion of foundation parameters caused a reduction in variation of non-dimensional axial and transverse shear stresses through dimensionless thickness.

\section{Nomenclature}

$$
\begin{aligned}
& x, y, z \\
& b, h, L \\
& E_{m}, E_{c} \\
& \nu_{m}, \nu_{c} \\
& K_{m}, K_{c} \\
& G_{m}, G_{c} \\
& K_{e}, G_{e}
\end{aligned}
$$
Young modulus of metal
constituents, respectively constituents, respectively constituents, respectively

Shear modulus of metal and ceramic constituents, respectively

Effective bulk and shear modulus,

$V_{m}, V_{c}$
$\lambda, \mu$
$u, w$
$u_{0}$
$w_{b}, w_{s}, w_{z}$
$u_{m}, w_{b m}, w_{s m}, w_{z m}$
$\bar{w}, \hat{w}$
$\bar{\sigma}_{x x}, \bar{\sigma}_{x z}$

$q$

$f, g$

$p$

$k_{w}, k_{p}$

$\bar{k}_{w}, \bar{k}_{p}$

$U, U_{f}, W$

$\delta_{i j}, e_{i p q}$

$\sigma_{i j}, \tau_{i j k}^{(1)}, m_{i j}^{s}, p_{i}$

$\varepsilon_{i j}, \gamma_{i}, \eta_{i j k}^{(1)}, \chi_{i j}^{s}$

$\eta_{i j k}^{s}$

$\eta_{i j k}$

$l_{0}, l_{1}, l_{2}$

$M, N, R, S$

$P$

$T$

\section{References}

1. Patocka, F., Schneidhofer, C., Dörr, N., et al. "Novel resonant MEMS sensor for the detection of particles with dielectric properties in aged lubricating oils", Sens. Actuators A Phys., 315, p. 112290 (2020).

2. Ulkir, O. "Design and fabrication of an electrothermal MEMS micro-actuator with 3D printing technology", Mater. Res. Express, 7, p. 075015 (2020).
Volume fraction of metal and ceramic constituents, respectively

Lame constants

Displacement of micro beam in $x$ and $z$ directions, respectively

Middle surface displacements in the $x$-direction

Bending, shear, and normal parts of transverse displacement, respectively

Dimensionless transverse deflection

Dimensionless axial and transverse

Distributed applied load

Shear deformation shape functions

Gradient power law index in the $z$ direction

Winkler and Pasternak stiffness parameters of the elastic foundation, respectively stiffness parameters, respectively Strain energy, strain energy of elastic foundation, and work of external applied forces, respectively

Kronecker delta and permutation symbol, respectively

Classical and higher order stresses tensors

Classical strain, dilatation gradient, deviatoric stretch gradient, and symmetric rotation gradient tensors, respectively

Symmetric part of second-order deformation gradient tensor Second-order deformation gradient tensor

Length scale parameters

Classical and non-classical forces and moment resultants

Dilatation resultants and moments

Deviatoric stretch resultants and moments stresses, respectively respectively 
3. Moutlana, M.K. and Adali, S. "Fundamental frequencies of a nano beam used for atomic force microscopy (AFM) in tapping mode", MRS Adv., 3, pp. 2617-2626 (2018).

4. Li, X.-F., Wang, B.-L., and Lee, K.Y. "Size effect in the mechanical response of nanobeams", J. of Adv. Res. in Mech. Eng., 1(1), pp. 4-16 (2010).

5. Dell'Isola, F., Andreaus, U., and Placidi, L. "At the origins and in the vanguard of peridynamics, nonlocal and higher-gradient continuum mechanics: An underestimated and still topical contribution of Gabrio Piola", Math. Mech. Solids, 20(8), pp. 887-928 (2015).

6. Cosserat, E. and Cosserat, F. "Théorie des corps déformables", Hermann, Paris, pp. 953-1173 (1909).

7. Thai, H-T., Vo, T.P., Nguyen, T.-K., et al. "A review of continuum mechanics models for size-dependent analysis of beams and plates", Compos. Struct., 177, pp. 196-219 (2017).

8. Mindlin, R.D. "Second gradient of strain and surfacetension in linear elasticity", Int. J. Solids Struct., 1(4), pp. 417-438 (1965).

9. Toupin, R. "Elastic materials with couple-stresses", Arch. Ration. Mech. Anal., 11(1), pp. 385-414 (1962).

10. Mindlin, R. and Tiersten, H. "Effects of couple-stresses in linear elasticity", Arch. Ration. Mech. Anal, 11, pp. 415-448 (1962).

11. Koiter, W.T. "Couple-stresses in the theory of elasticity, I \& II", Philos. Trans. R. Soc. Lond., B, 67, pp. 17-44 (1969).

12. Yang, F., Chong, A., Lam, D.C.C., et al. "Couple stress based strain gradient theory for elasticity", Int. J. Solids Struct., 39(10), pp. 2731-2743 (2002).

13. Fleck, N. and Hutchinson, J. "Strain gradient plasticity", Adv. Appl. Mech., 33, pp. 295-361 (1997).

14. Fleck, N. and Hutchinson, J. "A reformulation of strain gradient plasticity", J. Mech. Phys. Solids, 49(10), pp. 2245-2271 (2001).

15. Lam, D.C., Yang, F., Chong, A., et al. "Experiments and theory in strain gradient elasticity", J. Mech. Phys. Solids., 51(8), pp. 1477-1508 (2003).

16. Dal, H. "Analysis of gold micro-beams with modified strain gradient theory", Anadolu Üniversitesi Bilim Ve Teknoloji Dergisi A-Uygulamali Bilimler ve Mühendislik, 18(3), pp. 663-681 (2017).

17. Ashoori, A. and Mahmoodi, M. "A nonlinear thick plate formulation based on the modified strain gradient theory", Mech. Adv. Mater. Struct, 25(10), pp. 813819 (2018).

18. Chu, L., Dui, G., and Ju, C. "Flexoelectric effect on the bending and vibration responses of functionally graded piezoelectric nanobeams based on general modified strain gradient theory", Compos. Struct., 186, pp. 3949 (2018).

19. Thai, C.H., Ferreira, A., and Phung-Van, P. "Free vibration analysis of functionally graded anisotropic microplates using modified strain gradient theory", Eng. Anal. Bound. Elem., 117, pp. 284-298 (2020).
20. Farzam, A. and Hassani, B. "Size-dependent analysis of FG microplates with temperature-dependent material properties using modified strain gradient theory and isogeometric approach", Compos. B. Eng., 161, pp. 150-168 (2019).

21. Cornacchia, F., Fantuzzi, N., Luciano, R., et al. "Solution for cross-and angle-ply laminated Kirchhoff nano plates in bending using strain gradient theory", Compos. B. Eng., 173, p. 107006 (2019).

22. Thai, C.H., Ferreira, A., and Phung-Van, P. "A nonlocal strain gradient isogeometric model for free vibration and bending analyses of functionally graded plates", Compos. Struct., 251, pp. 112634 (2020).

23. Phung-Van, P., Ferreira, A., Nguyen-Xuan, H., et al. "An isogeometric approach for size-dependent geometrically nonlinear transient analysis of functionally graded nanoplates", Compos. B. Eng., 118, pp. 125134 (2017).

24. Phung-Van, P., Thai, C.H., Nguyen-Xuan, H., et al. "Porosity-dependent nonlinear transient responses of functionally graded nanoplates using isogeometric analysis", Compos. B. Eng., 164, pp. 215-225 (2019).

25. Phung-Van, P., Thai, C.H., Nguyen-Xuan, H., et al. "An isogeometric approach of static and free vibration analyses for porous FG nanoplates", Eur J Mech A Solids, 78, p. 103851 (2019).

26. Farzam, A. and Hassani, B. "Isogeometric analysis of in-plane functionally graded porous microplates using modified couple stress theory", Aerosp Sci Technol., 91, pp. 508-524 (2019).

27. Farzam, A. and Hassani, B. "Thermal and mechanical buckling analysis of FG carbon nanotube reinforced composite plates using modified couple stress theory and isogeometric approach", Compos. Struct., 206, pp. $774-790$ (2018).

28. Phung-Van, P., Thai, C.H., Wahab, M.A., et al. "Optimal design of FG sandwich nanoplates using sizedependent isogeometric analysis", Mech. Mater., 142, p. 103277 (2020).

29. Phung-Van, P., Ferreira, A., and Thai, C.H. "Computational optimization for porosity-dependent isogeometric analysis of functionally graded sandwich nanoplates", Compos. Struct., 239, pp. 112029 (2020).

30. Zhao, X., Zheng, S., and Li, Z. "Size-dependent nonlinear bending and vibration of flexoelectric nanobeam based on strain gradient theory", Smart Mater. Struct., 28, p. 075027 (2019).

31. Zanoosi, A.A.P. "Size dependent thermo mechanical free vibration analysis of functionally graded porous microbeams based on modified strain gradient theory", J. Braz. Soc. Mech. Sci., 42(5), p. 236 (2020).

32. Timoshenko, S.P. "On the correction for shear of the differential equation for transverse vibrations of prismatic bars", Philos. Mag., 41(245), pp. 744-746 (1921). 
33. Nam, V.H., Vinh, P.V., Chinh, N.V., et al. "A new beam model for simulation of the mechanical behaviour of variable thickness functionally graded material beams based on modified first order shear deformation theory", Materials, $12(3)$, p. 404 (2019).

34. Reddy, J. "A general non-linear third-order theory of plates with moderate thickness", Int. J. Nonlin. Mech., 25(6), pp. 677-686 (1990).

35. Ghugal, Y.M. and Sharma, R. "A hyperbolic shear deformation theory for flexure and vibration of thick isotropic beams", Int. J. Comput. Methods, 6(04), pp. 585-604 (2009).

36. Soldatos, K. "A transverse shear deformation theory for homogeneous monoclinic plates", Acta Mech., 94(3-4), pp. 195-220 (1992).

37. Ninh, D.G. and Bich, D.H. "Nonlinear thermal vibration of eccentrically stiffened ceramic-FGM-metal layer toroidal shell segments surrounded by elastic foundation", Thin-Walled Struct., 104, pp. 198-210 (2016).

38. Ninh, D.G. and Bich, D.H. "Nonlinear buckling of eccentrically stiffened functionally graded toroidal shell segments under torsional load surrounded by elastic foundation in thermal environment", Mech. Res. Commun., 72, pp. 1-15 (2016).

39. Carrera, E. "Theories and finite elements for multilayered plates and shells: a unified compact formulation with numerical assessment and benchmarking", Arch. Comput. Methods Eng. 10(3), pp. 215-296 (2003).

40. Demasi, L. " $\infty 6$ mixed plate theories based on the generalized unified formulation. Part I: Governing equations", Compos. Struct., 87(1), pp. 1-11 (2009).

41. Karamanlı, A. and Vo, T.P. "Size dependent bending analysis of two directional functionally graded microbeams via a quasi-3D theory and finite element method", Compos. B. Eng., 144, pp. 171-183 (2018).

42. Benahmed, A., Houari, M.S.A., Benyoucef, S., et al. "A novel quasi-3D hyperbolic shear deformation theory for functionally graded thick rectangular plates on elastic foundation", Geomech. Eng., 12(1), pp. 9-34 (2017).

43. Nguyen, T.-K., Vo, T.P., Nguyen, B.-D., et al. "An analytical solution for buckling and vibration analysis of functionally graded sandwich beams using a quasi3D shear deformation theory", Compos. Struct., 156, pp. 238-252 (2016).

44. Farzam, A. and Hassani, B. "A new efficient shear deformation theory for FG plates with in-plane and through-thickness stiffness variations using isogeometric approach", Mech. Adv. Mater. Struct, , 26, pp. 512525 (2019).

45. Farzam-Rad, S.A., Hassani, B., and Karamodin, A. "Isogeometric analysis of functionally graded plates using a new quasi-3D shear deformation theory based on physical neutral surface", Compos. B. Eng., 108, pp. 174-189 (2017).
46. Thai, C.H., Ferreira, A., Tran, T., et al. "A sizedependent quasi-3D isogeometric model for functionally graded graphene platelet-reinforced composite microplates based on the modified couple stress theory", Compos. Struct., 234, p. 111695 (2020).

47. Winkler, E. "Theory of elasticity and strength", Dominicus Prague, Czechoslovakia (1867).

48. Pasternak, P. "On a new method of analysis of an elastic foundation by means of two foundation constants" (in Russian), Gosudarstvennoe Izdatelstvo Literaturi po Stroitelstvu I Arkhitekture, USSR (1954).

49. Atmane, H.A., Tounsi, A., and Bernard, F. "Effect of thickness stretching and porosity on mechanical response of a functionally graded beams resting on elastic foundations", Int. J. Mech. Mater. Des., 13(1), pp. 71-84 (2017).

50. Lee, W.-H., Han, S.-C., Park, W.-T. "A refined higher order shear and normal deformation theory for E-, P-, and S-FGM plates on Pasternak elastic foundation", Compos. Struct., 122, pp. 330-342 (2015).

51. Li, Q., Wu, D., Gao, W., et al. "Size-dependent instability of organic solar cell resting on WinklerPasternak elastic foundation based on the modified strain gradient theory", Int. J. Mech. Sci., 177, p. 105306 (2020).

52. Ninh, D.G., Tien, N.D., Hoang, V.N.V., et al. "Vibration of cylindrical shells made of three layers W-Cu composite containing heavy water using Flügge-Lur'eBryrne theory", Thin-Walled Struct., 146, p. 106414 (2020).

53. Zeighampour, H., Beni, Y.T., and Dehkordi, M.B. "Wave propagation in viscoelastic thin cylindrical nanoshell resting on a visco-Pasternak foundation based on nonlocal strain gradient theory", ThinWalled Struct., 122, pp. 378-386 (2018).

54. Cao, C.-Y. and Zhong, Y. "Dynamic response of a beam on a Pasternak foundation and under a moving load", J. of Chongqing Univ., 7(4), pp. 311-316 (2008).

55. Kural, S. and Özkaya, E. "Size-dependent vibrations of a micro beam conveying fluid and resting on an elastic foundation", J. Vib. Control, 23(7), pp. 11061114 (2017).

56. Eyebe, G., Betchewe, G., Mohamadou, A., et al. "Nonlinear vibration of a nonlocal nanobeam resting on fractional-order viscoelastic Pasternak foundations", Fractal Fract., 2(3), p. 21 (2018).

57. Bich, D.H. and Ninh, D.G. "Research on dynamical buckling of imperfect stiffened three-layered toroidal shell segments containing fluid under mechanical loads", Acta Mech., 228, pp. 711-730 (2017).

58. Bich, D.H. Ninh, D.G. "An analytical approach: Nonlinear vibration of imperfect stiffened FGM sandwich toroidal shell segments containing fluid under external thermo-mechanical loads", Compos. Struct, 162, pp. 164-181 (2017). 
59. Bich, D.H. and Ninh, D.G. "Post-buckling of sigmoidfunctionally graded material toroidal shell segment surrounded by an elastic foundation under thermomechanical loads", Compos. Struct., 138, pp. 253-263 (2016).

60. El Meiche, N., Tounsi, A., Ziane, N., et al. "A new hyperbolic shear deformation theory for buckling and vibration of functionally graded sandwich plate", Int. J. Mech. Sci., 53(4), pp. 237-247 (2011).

61. Mori, T. and Tanaka, K. "Average stress in matrix and average elastic energy of materials with misfitting inclusions", Acta Metall., 21(5), pp. 571-574 (1973).

62. Trinh, L.C., Nguyen, H.X., Vo, T.P., et al. "Sizedependent behavior of functionally graded microbeams using various shear deformation theories based on the modified couple stress theory", Compos. Struct., 154, pp. 556-572 (2016).

63. Ke, L.-L. and Wang, Y.-S. "Size effect on dynamic stability of functionally graded microbeams based on a modified couple stress theory", Compos. Struct., 93(2), pp. $342-350$ (2011).

\section{Biographies}

Mohammad Gholami is currently a $\mathrm{PhD}$ candidate at the School of Mechanical Engineering at Iran University of Science and Technology, Tehran, Iran. He received his MS from Shiraz University of Technology. His research interests include size-dependent behavior, static and dynamic analyses of composite structures, computational mechanics, and viscoelastic time-dependent materials. He has published several papers in reputable journals and international conference proceedings.

Mansour Alizadeh received his BS from Frankfurt University, MS from Amirkabir University of Technology, and PhD from Technical University of Berlin. He is currently an Assistant Professor in Mechanical Engineering at Iran University of Science and Technology, Tehran, Iran. His main research interests include continuum mechanics, size-dependent effect, and nonlinear mechanics. He is the author and coauthor of many technical papers in these fields. 